\title{
多烷基苯磺酸钠水溶液的表 面性质
}

\author{
姜小明张路安静仪赵濉俞稼锯
}

(中国科学院理化技术研究所, 北京 100101)

\begin{abstract}
摘要 研究了多烷基苯磺酸钠的结构, 特别是侧链碳原子数的增加, 对其表面活性的影响, 并与其它烷基苯磺酸 钠进行了比较. 结果表明, 随着苯环上侧链碳原子数的增加, 多烷基苯磺酸钠的临界胶束浓度 $(\mathrm{cmc})$ 降低, 但侧链 上 $\mathrm{CH}_{2}$ 降低 $\mathrm{cmc}$ 的程度远小于主链上 $\mathrm{CH}_{2}$ 的作用. 当侧链碳原子数增加时, 多烷基苯磺酸钠的饱和吸附量 $\left(\Gamma_{\max }\right)$ 降低, 表现出与主链不同的变化规律. 从多烷基苯磺酸钠的结构解释了 $\mathrm{cmc}$ 和 $\Gamma_{\text {max }}$ 的变化规律.
\end{abstract}

关键词：多烷基苯磺酸钠，分子结构，临界胶束浓度，饱和吸附量 中图分类号： 0647.3

表面活性剂的化学结构与表面性质密切相关, 例如疏水基长度的变化、分支及极性基位置对表面 性质都有重要影响. 有关烷基苯磺酸钠分子结构与 性质关系的研究已有大量报道 ${ }^{[1-2]}$, 但均集中在主链 的变化 ${ }^{[3]}$ (如: 改变链长, 是否支化等), 而对于苯环上 短侧链的变化如何影响烷基苯磺酸钠的表面性质的 报道还很少. 这主要是烷基苯磺酸钠纯化困难, 含短 碳链取代基的多取代的烷基苯磺酸钠不易合成. 本 文用自制的高纯度多烷基苯磺酸钠系列, 研究了侧 链的变化对烷基苯磺酸钠表面性质的影响.

\section{1 实验部分}

\section{1 试剂与仪器}

多种多烷基苯磺酸钠, 自制 ${ }^{[4]}$, 其分子结构经IR 和 ${ }^{1} \mathrm{H}$ NMR 确定, 纯度大于 $98.0 \%$, 各烷基苯磺酸钠 的克拉夫特点 (Krafft point)均在 $0 \sim 20{ }^{\circ} \mathrm{C}$, 其结构及 编号见图 1 ; 氯化钠为分析纯; 实验用水为二次蒸馏 水.

表面张力测定用 DATAPHYSICS dcat21 接触 角/表面张力测量仪; 水温 $\left(30{ }^{\circ} \mathrm{C}\right)$ 用 $\mathrm{BX}-101$ 型超级 恒温槽控制.

\section{2 实验方法}

配制一系列不同浓度的多烷基苯磺酸钠水溶 液和多烷基苯磺酸钠的 $\mathrm{NaCl}$ 水溶液 $(c(\mathrm{NaCl})=0.171$ $\left.\mathrm{mol} \cdot \mathrm{L}^{-1}\right)$, 静置 $8 \mathrm{~h}, 30{ }^{\circ} \mathrm{C}$ 下用 Wilhelmy-plate 法测定
平衡表面张力.

\section{2 理论计算}

烷基苯磺酸钠的饱和吸附量 $\left(\Gamma_{\max }\right)$ 由 Gibbs 吸 附公式 ${ }^{[5]}$ 计算:

$$
\Gamma_{\max }=[-1 /(2.303 n R T)] \times[\mathrm{d} \gamma / \mathrm{d} \lg \alpha]_{\max }
$$

式中 $\alpha$ 为烷基苯磺酸钠在溶液中的活度; $R$ 是气体 常数; $T$ 是绝对温度; $n$ 为疏水碳氢链的碳原子数; $\gamma$ 为烷基苯磺酸钠水溶液的表面张力.

$A_{\text {min }}$ 为饱和吸附时烷基苯磺酸钠分子在溶液表 面的极限占有面积, 可由公式(2)计算 ${ }^{\left[{ }^{[}\right]}$:

$$
A_{\min }=1 /\left[N \Gamma_{\max }\right]
$$

$N$ 是阿佛加得罗常数,标准吸附自由能 $\left(\Delta G_{\mathrm{ad}}^{0}\right)$ 可 由公式(3)计算 ${ }^{[4]}$ :<smiles>[R]c1cc([R9])c([N+](=O)[O-])cc1C([R])[R]</smiles>

5,6-1,1: $\mathrm{R}_{1}=\mathrm{R}_{2}=\mathrm{CH}_{3}, \quad \mathrm{R}_{3}=\mathrm{C}_{5} \mathrm{H}_{11}, \mathrm{R}_{1}=\mathrm{C}_{6} \mathrm{H}_{13}$ 5,6-3,3: $\mathrm{R}_{1}=\mathrm{R}_{2}=i-\mathrm{C}_{3} \mathrm{H}_{7}, \quad \mathrm{R}_{3}=\mathrm{C}_{5} \mathrm{H}_{11}, \mathrm{R}_{4}=\mathrm{C}_{6} \mathrm{H}_{13}$ 5,6-2,0: $\mathrm{R}_{1}^{=} \mathrm{C}_{2} \mathrm{H}_{5}, \mathrm{R}_{2}=\mathrm{H}, \mathrm{R}_{3}=\mathrm{C}_{5} \mathrm{H}_{11}, \mathrm{R}_{4}=\mathrm{C}_{6} \mathrm{H}_{13}$

图 1 多烷基苯磺酸钠的分子结构及编号

Fig.1 The molecular structures and serial numbers of sodium alkylbenzensulfonates

2005-04-07 收到初稿, 2005-07-07 收到修改稿. 联系人:安静仪(E-mail:jyan@ipc.ac.cn; Tel:010-64888165). “国家重点基础研究发展 规划项目(G19990225)资助 


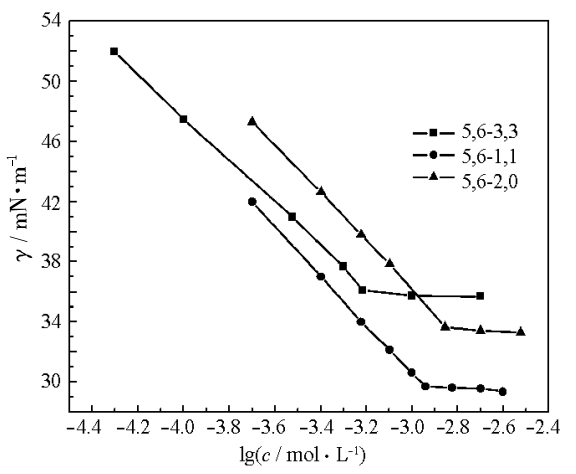

图 2 多烷基苯磺酸钠在纯水中的 $\gamma-\lg c$ 曲线

Fig.2 Surface tension $v s \lg c$ of sodium alkylbenzensulfonates in pure water

$$
\Delta G_{\mathrm{ad}}^{0}=2.303 R T \lg \alpha_{\mathrm{R}^{-}, \mathrm{cmc}}-0.602 \pi_{\mathrm{cmc}} A_{\text {min }}
$$

式中 $\alpha_{R^{-}, \mathrm{cmc}}$ 为临界胶束浓度时表面活性剂阴离子的 活度, $\pi_{\mathrm{cmc}}$ 为临界胶束浓度时表面活性剂溶液的表 面压. $\mathrm{pC}_{20}$ 是将水溶液表面张力降低 $20 \mathrm{mN} \cdot \mathrm{m}^{-1}$ 所 需表面活性剂浓度的负对数, 可由公式(4)计算 ${ }^{[5]}$ :

$$
\mathrm{pC}_{20}=\left[\left(\gamma_{0}-20-\gamma_{\mathrm{cmc}}\right) /\left(2.303 n R \Gamma_{\max }\right)\right]-\lg \alpha_{\mathrm{cmc}}
$$

式中 $\gamma_{0}$ 为纯水的表面张力, $\gamma_{\mathrm{cmc}}$ 为临界胶束浓度时 表面活性剂溶液的表面张力, $\alpha_{\mathrm{cmc}}$ 为临界胶束浓度 时表面活性剂的活度.

\section{3 结果与讨论}

图 2 和图 3 分别为 $30{ }^{\circ} \mathrm{C}$ 时多烷基苯磺酸钠在 纯水及 $0.171 \mathrm{~mol} \cdot \mathrm{L}^{-1} \mathrm{NaCl}$ 水溶液中表面张力随浓 度变化的曲线. 由 $\gamma-\lg c$ 曲线计算各多烷基苯磺酸 钠的表面性质数据, 列于表 1 和表 2 .

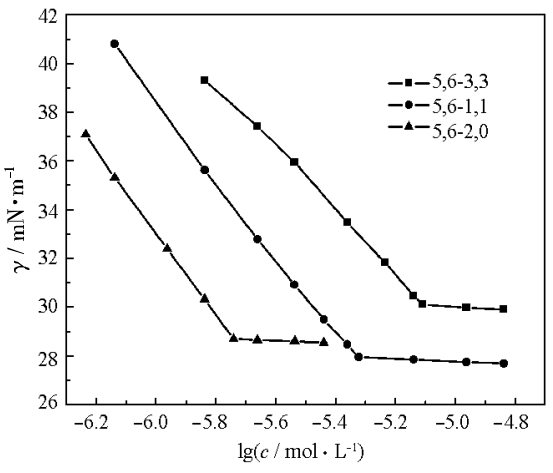

图 3 多烷基苯磺酸钠在氯化钠溶液中的 $\gamma-\lg c$ 曲线

Fig.3 Surface tension $v s \lg c$ of sodium alkylbenzensulfonates in $0.171 \mathrm{~mol} \cdot \mathrm{L}^{-1} \mathrm{NaCl}$ solution

\section{1 支链烷基苯磺酸钠的结构与 $\mathrm{cmc}$ 的关系}

临界胶束浓度 $(\mathrm{cmc})$ 是表面活性剂的重要性质 之一. 为了研究烷基苯磺酸钠的主链、侧链对 $\mathrm{cmc}$ 的影响, 表 3 列出了单取代烷基苯磺酸钠、单取代烷 基荟磺酸钠、三取代烷基苯磺酸钠(分子结构见图 4) 及多取代烷基苯磺酸钠的 $\mathrm{cmc}$ 数据.

从表 3 可知, 碳氢主链碳原子数的增加和短侧 链中碳原子数的增加对 $\mathrm{cmc}$ 的降低程度是不同的. I-III 组考查芳环上碳氢主链碳原子数的增大对 $\mathrm{cmc}$ 的影响. 其中 $1 、 2 、 3$ 为单取代烷基苯磺酸钠, 碳氢主 链每增加两个碳原子, $\mathrm{cmc}$ 约为原值的 $1 / 4 ; 4 、 5 、 6$ 为单取代烷基荟磺酸钠, 碳氢主链每增加两个碳, $\mathrm{cmc}$ 约为原值的 $1 / 4$. 大体上计算, 碳氢主链平均每 增加一个碳, $\mathrm{cmc}$ 降低一半. 第 III 组 7、8、9 为三烷基 苯磺酸钠, 碳氢主链每增加一个碳, $\mathrm{cmc}$ 降低一半.

表 1 多烷基苯磺酸钠在水溶液中的表面性质参数

\begin{tabular}{|c|c|c|c|c|c|c|c|}
\hline Surfactants & $\frac{10^{-3} \mathrm{cmc}}{\mathrm{mol} \cdot \mathrm{L}^{-1}}$ & $\frac{10^{10} \Gamma_{\max }}{\mathrm{mol} \cdot \mathrm{cm}^{-2}}$ & $\begin{array}{l}A_{\min } \\
\mathrm{nm}^{2}\end{array}$ & $\frac{\gamma_{\mathrm{cmc}}}{\mathrm{mN} \cdot \mathrm{m}^{-1}}$ & $\frac{\pi_{\mathrm{cmc}}}{\mathrm{mN} \cdot \mathrm{m}^{-1}}$ & $\mathrm{pC}_{20}$ & $\frac{\Delta G_{\mathrm{ad}}^{0}}{\mathrm{~kJ} \cdot \mathrm{mol}^{-1}}$ \\
\hline $5,6-3,3$ & 0.63 & 1.23 & 1.35 & 35.91 & 35.09 & 4.26 & -47.06 \\
\hline $5,6-1,1$ & 1.15 & 1.44 & 1.18 & 29.61 & 41.49 & 4.23 & -46.52 \\
\hline $5,6-2,0$ & 1.40 & 1.38 & 1.20 & 33.66 & 37.54 & 3.95 & -43.66 \\
\hline
\end{tabular}

Table 1 Surfacial properties of sodium alkylbenzensulfonates in pure water

cmc is the critical micelle concentration; $\Gamma_{\max }$ is the surface excess concentration at surface saturation. $A_{\min }$ is minimum areas. $\gamma_{\mathrm{cmc}}$ is the surface tension at the cmc; $\pi_{\mathrm{cmc}}$ is the effectiveness of a surfactant in reduing surface tension. $\mathrm{pC}_{20}$ is the efficiency of a surfactant in reduing surface tension. $\Delta G_{\mathrm{ad}}^{0}$ is the standard free energy change upon adsorption.

表 2 多烷基苯磺酸钠在氯化钠溶液中的表面性质参数

\begin{tabular}{|c|c|c|c|c|c|c|c|}
\hline \multirow{2}{*}{ Surfactants } & $10^{-3} \mathrm{cmc}$ & $10^{10} \Gamma_{\max }$ & $A_{\min }$ & $\gamma_{\mathrm{cmc}}$ & $\pi_{\mathrm{cmc}}$ & \multirow{2}{*}{$\mathrm{pC}_{20}$} & $\Delta G_{\mathrm{ad}}^{0}$ \\
\hline & $\mathrm{mol} \cdot \mathrm{L}^{-1}$ & $\mathrm{~mol} \cdot \mathrm{cm}^{-2}$ & $\mathrm{~nm}^{2}$ & $\mathrm{mN} \cdot \mathrm{m}^{-1}$ & $\mathrm{mN} \cdot \mathrm{m}^{-1}$ & & $\mathrm{~kJ} \cdot \mathrm{mol}^{-1}$ \\
\hline $5,6-3,3$ & 0.110 & 2.21 & 0.75 & 30.12 & 41.08 & 5.61 & -47.86 \\
\hline $5,6-1,1$ & 0.065 & 2.84 & 0.58 & 27.95 & 43.25 & 5.47 & -45.74 \\
\hline $5,6-2,0$ & 0.025 & 2.90 & 0.57 & 28.70 & 42.50 & 5.85 & -47.63 \\
\hline
\end{tabular}

Table 2 Surfacial properties of sodium alkylbenzensulfonates in $0.171 \mathrm{~mol} \cdot \mathrm{L}^{-1} \mathrm{NaCl}$ solution 
表 3 不同结构烷基芳基磺酸钠在 $30^{\circ} \mathrm{C}$ 水溶液中的临界胶束浓度

Table $3 \mathrm{cmc}$ of the different sodium alkylbenzensulfonates in aqueous solution $\left(30{ }^{\circ} \mathrm{C}\right)$

\begin{tabular}{ccccc}
\hline Group & No. & Surfactants & $10^{-3} \mathrm{cmc}\left(\mathrm{mol} \cdot \mathrm{L}^{-1}\right)$ & $B$ \\
\hline I & 1 & $p-2-\mathrm{C}_{14} \mathrm{H}_{29} \mathrm{C}_{6} \mathrm{H}_{4} \mathrm{SO}_{3} \mathrm{Na}$ & $1.534^{[6]}$ & 0.28 \\
& 2 & $p-2-\mathrm{C}_{16} \mathrm{H}_{33} \mathrm{C}_{6} \mathrm{H}_{4} \mathrm{SO}_{3} \mathrm{Na}$ & $0.438^{[6]}$ & \\
& 3 & $p-2-\mathrm{C}_{18} \mathrm{H}_{37} \mathrm{C}_{6} \mathrm{H}_{4} \mathrm{SO}_{3} \mathrm{Na}$ & $0.112^{[6]}$ & \\
II & 4 & $1-\left(n-\mathrm{C}_{6} \mathrm{H}_{13}\right)-4-\mathrm{C}_{10} \mathrm{H}_{6} \mathrm{SO}_{3} \mathrm{Na}$ & $10^{[7]}$ & 0.28 \\
& 5 & $1-\left(n-\mathrm{C}_{8} \mathrm{H}_{17}\right)-4-\mathrm{C}_{10} \mathrm{H}_{6} \mathrm{SO}_{3} \mathrm{Na}$ & $2.4^{[7]}$ & \\
& 6 & $1-\left(n-\mathrm{C}_{10} \mathrm{H}_{21}\right)-4-\mathrm{C}_{10} \mathrm{H}_{6} \mathrm{SO}_{3} \mathrm{Na}$ & $0.80^{[7]}$ & \\
III & 7 & $3,4-3,3\left(N_{\mathrm{C}}=14\right)$ & $4.0^{[4]}$ & 0.30 \\
& 8 & $4,4-3,3\left(N_{\mathrm{C}}=15\right)$ & $2.0^{[4]}$ & \\
& 9 & $4,5-3,3\left(N_{\mathrm{C}}=16\right)$ & $1.0^{[4]}$ & \\
IV & 10 & $p-5-\mathrm{C}_{12} \mathrm{H}_{25} \mathrm{C}_{6} \mathrm{H}_{4} \mathrm{SO}_{3} \mathrm{Na}\left(N_{\mathrm{C}}=12\right)$ & $1.63^{[8]}$ & 0.068 \\
& 11 & $5,6-1,1\left(N_{\mathrm{C}}=14\right)$ & 1.15 & \\
& 12 & $5,6-3,3\left(N_{\mathrm{C}}=18\right)$ & 0.68 & \\
\hline
\end{tabular}

(1) $N_{\mathrm{C}}$ is the total carbon number of alkyl chain of an alkylbenzensulfonate.

(2) Although surfactants in group III and IV are all alkylbenzensulfonates, the position of alkyl chain at benzene ring is different, so the cmc in group III and IV can't be compared.

(3) $B$ is a constant for the ionic type of alkylbenzensulfonate.

根据表面活性剂作用原理，离子型表面活性剂 碳氢链的碳原子数在 8 16 的范围时, $\mathrm{cmc}$ 随碳原子 数变化呈现一定的规律, 即在同系物中每增加一个 碳原子, cmc 下降一半 ${ }^{[9]}$. 在表 3 中, 无论单取代烷基 苯磺酸钠、单取代烷基芸磺酸钠, 还是三烷基苯磺酸 钠, 碳氢主链碳原子数的变化引起的 $\mathrm{cmc}$ 的变化基 本符合这一规律.

但当多烷基苯磺酸钠 11 和 12 (表 3 第 IV 组)的 短侧链碳原子数变化时, 引起 $\mathrm{cmc}$ 的变化与上述规 律差异很大. 比较 $10 、 11$ 和 12 , 它们主链碳原子数 相同, 10 和 11 的主链支化位置只有一个碳原子之 差, 有一定的可比性, 11 比 10 短侧链增加了两个碳 原子, 但 11 的临界胶束浓度的降低不足 10 的一半. 11 和 12 主链分支的位置完全相同, 12 比 11 侧链增<smiles>[R]c1cc(C([R])[R])cc([NH2+][O-])c1[R]</smiles>

3,4-3,3: $\mathrm{R}_{1}=i-\mathrm{C}_{3} \mathrm{H}_{7}, \quad \mathrm{R}_{2}=n-\mathrm{C}_{3} \mathrm{H}_{7}, \quad \mathrm{R}_{3}=\mathrm{C}_{3} \mathrm{H}_{7}, \quad \mathrm{R}_{4}=\mathrm{C}_{4} \mathrm{H}_{9}$ 4,4-3,3: $\mathrm{R}_{1}=i-\mathrm{C}_{3} \mathrm{H}_{7}, \mathrm{R}_{2}=n-\mathrm{C}_{3} \mathrm{H}_{7}, \mathrm{R}_{3}=\mathrm{C}_{4} \mathrm{H}_{9}, \quad \mathrm{R}_{4}=\mathrm{C}_{4} \mathrm{H}_{9}$ 4,5-3,3: $\mathrm{R}_{1}=i-\mathrm{C}_{3} \mathrm{H}_{7}, \mathrm{R}_{2}=n-\mathrm{C}_{3} \mathrm{H}_{7}, \mathrm{R}_{3}=\mathrm{C}_{4} \mathrm{H}_{9}, \mathrm{R}_{4}=\mathrm{C}_{5} \mathrm{H}_{11}$

图 4 三烷基苯磺酸钠(表 3 中第 III 组)的分子结构图

Fig.4 The molecular structure of sodium trialkylbenzensulfonates (Table 3, group III)
加了四个碳原子, $\mathrm{cmc}$ 的降低仍不足一半. 上述结果 表明, 主链与短侧链碳原子数的增大, 对降低 $\mathrm{cmc}$ 的程度是不同的. 短侧链降低 $\mathrm{cmc}$ 的能力, 远小于 主链. 这说明多烷基苯磺酸钠在形成胶团的过程中, 短侧链的疏水相互作用不如主链明显.

直链离子型表面活性剂同系物满足公式:

$$
\lg \mathrm{cmc}=A-B n
$$

$A 、 B$ 均为经验常数, 其大小分别代表极性基和疏水 基对表面活性剂形成胶团能力的影响的强弱. 表 3 列出不同支链烷基苯磺酸钠的经验常数 $B$ 的值. 从 表中可以看出 I、II、III 的 $B$ 值相近, 都在 0.30 左右, 而 IV 的 $B$ 值则小得多. 这说明多烷基苯磺酸钠形 成胶团时, 侧链的影响不如主链影响大.

Caponetti 等 ${ }^{[10]}$ 用小角度中子衍射法(SANS)研 究支链烷基苯磺酸钠胶团结构时, 提出了胶团的结 构模型. 在该模型中, 侧链位于胶团的栅栏层附近. 从胶团形成过程来看,一方面, 短侧链的增加, 使表 面活性剂分子间疏水相互作用增强, cmc 降低; 另一 方面, 短侧链的增加使表面活性剂分子形成胶束时 空间阻碍增大, cmc 升高. 这两个相互竞争的因素决 定了短侧链降低 $\mathrm{cmc}$ 的程度没有主链明显.

从胶团形成的热力学来看, $B$ 值反映疏水基中 一个次甲基由水环境转移到胶团时标准 Gibbs 自由 能的改变量,一般表面活性剂的 $B$ 值与次甲基由水 环境转移到碳氢环境时自由能的改变量相符合 ${ }^{[1]}$. 而多取代烷基苯磺酸盐邻位的侧链在形成胶团时并 
表 4 烷基苯磺酸钠在水溶液中的 $\boldsymbol{\Gamma}_{\text {max }}$

Tables $4 \quad \Gamma_{\max }$ of sodium alkylbenzensulfonates in water

\begin{tabular}{ccccc}
\hline Group & No. & Surfactants & $T /{ }^{\circ} \mathrm{C}$ & $\frac{10^{10} \Gamma_{\text {max }}}{\mathrm{mol} \cdot \mathrm{cm}^{-2}}$ \\
\hline I & 1 & $\mathrm{C}_{9} \mathrm{H}_{19} \mathrm{C}_{6} \mathrm{H}_{4} \mathrm{SO}_{3} \mathrm{Na}$ & 75 & $1.8^{[6]}$ \\
& 2 & $\mathrm{C}_{10} \mathrm{H}_{21} \mathrm{C}_{6} \mathrm{H}_{4} \mathrm{SO}_{3} \mathrm{Na}$ & 75 & $2.1^{[6]}$ \\
& 3 & $\mathrm{C}_{12} \mathrm{H}_{25} \mathrm{C}_{6} \mathrm{H}_{4} \mathrm{SO}_{3} \mathrm{Na}$ & 75 & $2.8^{[6]}$ \\
II & 4 & $4,7-1\left(N_{\mathrm{C}}=13\right)$ & 30 & $1.45^{[8]}$ \\
& 5 & $6,7-1\left(N_{\mathrm{C}}=15\right)$ & 30 & $1.46^{[8]}$ \\
& 6 & $8,7-1\left(N_{\mathrm{C}}=17\right)$ & 30 & $1.87^{[[8]}$ \\
III & 7 & $p-5-\mathrm{C}_{12} \mathrm{H}_{25} \mathrm{C}_{6} \mathrm{H}_{4} \mathrm{SO}_{3} \mathrm{Na}\left(N_{\mathrm{C}}=12\right)$ & 30 & $1.50^{[10]}$ \\
& 8 & $5,6-1,1\left(N_{\mathrm{C}}=14\right)$ & 30 & 1.44 \\
& 9 & $5,6-3,3\left(N_{\mathrm{C}}=18\right)$ & 30 & 1.23 \\
\hline
\end{tabular}

未转移到完全的碳氢环境中, 导致 $B$ 值的降低.

\section{2 多烷基苯磺酸钠的结构与 $\boldsymbol{\Gamma}_{\text {max }}$ 的关系}

$\Gamma_{\text {max }}$ 是衡量表面活性剂吸附能力的重要参数. 为了研究烷基苯磺酸钠中碳氢主链和短侧链对 $\Gamma_{\text {max }}$ 的影响, 表 4 列出了单取代直链烷基苯磺酸钠(I), 二取代支链烷基苯磺酸钠(II)及多取代支链烷基苯 磺酸钠(III)的 $\Gamma_{\text {max }}$. 第 I、II 组研究碳氢主链对于 $\Gamma_{\text {max }}$ 的影响, 第 III 组研究短侧链对 $\Gamma_{\text {max }}$ 的影响.

从表 4 中可知, 对于烷基芳基磺酸钠, 碳氢主链 和短侧链的碳原子数增加对 $\Gamma_{\text {max }}$ 有不同影响. $1 、 2 、$ 3 为单取代烷基苯磺酸钠, 随着主链碳原子数的增 加, $\Gamma_{\text {max }}$ 也随之略有增加; 4、5、6 为二取代支链烷基 苯磺酸钠, $\Gamma_{\text {max }}$ 也是随主链碳原子数的增加而增大. $7 、 8 、 9$ 为多烷基苯磺酸钠, $\Gamma_{\text {max }}$ 随着侧链碳原子数 的增加反而降低.

考查多烷基苯磺酸钠的 $\Gamma_{\text {max }}$ 时, 主要考虑界面 化学势与体相化学势的差值、分子的独占面积(即不 允许其它分子进人的面积, 由表面活性剂分子大小 及其在界面上的排列方式决定)及离子头之间的排 斥力的影响.

对于第 I 组和第 II 组烷基苯磺酸钠, 随着碳氢 主链的增加, 疏水性增强, 界面化学势与体相化学势 的差值增大, 因此 $\Gamma_{\text {max }}$ 增大. 对第 III 组烷基苯磺酸 钠, 其主链碳原子数保持不变, $\Gamma_{\text {max }}$ 受侧链的影响: 短侧链碳原子数的增加使分子代表性面积增大, 导 致 $\Gamma_{\text {max }}$ 降低.

\section{3 多烷基苯磺酸钠的结构与 $\mathrm{pC}_{20} 、 \Delta G_{\mathrm{ad}}^{0}$ 的关系}

$\mathrm{pC}_{20}$ 可以作为表面活性剂降低表面张力效率的 量度. 从表 5 可知, 多烷基苯磺酸钠的 $\mathrm{pC}_{20}$ 随着侧 链碳原子数的增加而增加.

$\Delta G_{\mathrm{ad}}^{0}$ 是表面活性剂的标准吸附自由能. 从同系
表 5 烷基苯磺酸钠在水溶液中的 $\mathrm{pC}_{20} 、 \Delta \boldsymbol{G}_{\mathrm{ad}}^{0}$

Table $5 \mathrm{pC}_{20}$ and $\Delta G_{\mathrm{ad}}^{0}$ of sodium alkylbenzensulfonates in water

\begin{tabular}{ccc}
\hline Surfactants & $\mathrm{pC}_{20}$ & $\Delta G_{\mathrm{ad}}^{0}\left(\mathrm{~kJ} \cdot \mathrm{mol}^{-1}\right)$ \\
\hline$p-5-\mathrm{C}_{12} \mathrm{H}_{25} \mathrm{C}_{6} \mathrm{H}_{4} \mathrm{SO}_{3} \mathrm{Na}$ & 3.99 & -43.20 \\
$5,6-1,1$ & 4.23 & -46.52 \\
$5,6-3,3$ & 4.26 & -47.06 \\
\hline
\end{tabular}

物的吸附标准自由能可推算出每增加一个次甲基对 自由能的贡献约为 $3.0 \sim 3.5 \mathrm{~kJ} \cdot \mathrm{mol}^{-1}[9]$. 从表 5 可知, $\Delta G_{\text {ad }}^{0}$ 随着侧链碳原子数的增加而降低, 说明侧链碳 原子数的增加使表面活性剂疏水性增强. 但侧链增 加 4 个碳原子, 自由能的改变量仅为 $0.5 \mathrm{~kJ} \cdot \mathrm{mol}^{-1}$ 左 右. 这是由于多取代烷基苯磺酸盐在表面上排列时, 侧链大部分处于水环境中造成的.

\section{4 氯化钠对多烷基苯磺酸钠性质的影响}

从表 1 和表 2 可知, 加人氯化钠后, 多烷基苯磺 酸钠 5,6-3,3、5,6-1,1 和 5,6-2,0 的 $\mathrm{cmc}$ 与纯水中的 $\mathrm{cmc}$ 相比, 分别降低了 5.7、17.6 和 56 倍. 这说明多 烷基苯磺酸钠因侧链的不同而对盐的敏感性是不同 的. 加人氯化钠后, 电性排斥变弱, 多烷基苯磺酸钠 的空间阻碍成为影响 $\mathrm{cmc}$ 的重要因素. 多烷基苯磺 酸钠 5,6-3,3、5,6-1,1 和 5,6-2,0 的空间阻碍依次降 低, 胶团越容易形成, 因此 $\mathrm{cmc}$ 降低程度增大, 对盐 的敏感性增强.

从表 1 和表 2 还可看出, 加人氯化钠后, 5,63,3、5,6-1,1 和 5,6-2,0 的 $\Gamma_{\text {max }} \mathrm{pC}_{20}$ 都增大了. 说明无 机盐的加人, 提高了表面活性剂降低表面张力的效 率. 加人无机盐, 对 $\Delta G_{\text {ad }}^{0}$ 影响不大.

\section{4 结 论}

在多烷基苯磺酸钠溶液中, 随着短侧链的碳原 子数的增加, $\mathrm{cmc}$ 降低, 但降低的程度远小于主链对 $\mathrm{cmc}$ 的降低程度. 短侧链碳原子数的增加, 使 $\Gamma_{\text {max }}$ 降 低, 表现出与主链不同的变化规律. 短侧链碳原子数 的增加, 使 $\mathrm{pC}_{20}$ 增大, $\Delta G_{\mathrm{ad}}^{0}$ 更负. 加氯化钠后, $\mathrm{pC}_{20}$ 增大, 但对 $\Delta G_{\text {ad }}^{0}$ 影响不大. 从多烷基苯磺酸钠的结 构来看, 较大的空间位阻和分子独占面积是造成上 述实验结果的主要原因.

\section{References}

1 Tsubone, K.; Rosen, M. J. J. Colloid and Interface Sci., 2001, 244 (2): 394

2 Rosen, M. J.; Cohen, A.W.; Dahanayake, M. D.; Hua, X. Y. J. Phys. 
Chem., 1982, 86: 541

3 Wang, L.; Zhang, L.; Chu, Y. P.; Zhao, S.; Yu, J. Y. Acta Phys.-Chim. Sin., 2004, 20(12): 1451 [王 琳, 张 路, 楚艳苹, 赵 濉, 俞稼镛. 物理化学学报(Wuli Huaxue Xuebao), 2004, 20(12): 1451]

4 Jiang, X. M.; Xu, Z. C.; An, J. Y.; Zhao, S.; Yu, J. Y. Fine Chemicals, 2005, 21: 808 [姜小明, 徐志成, 安静仪, 赵 㴧, 俞 稼镛. 精细化工(Jingxi Huagong), 2005, 21: 808]

5 Dahanayake, M. D.; Coben, A. W.; Rosen, M. J. Phys. Chem., 1986, 90: 2413

6 Shi, F. Q.; Zhang, L.; An, J. Y.; Zhao, S.; Yu, J. Y. Photographic Sci.\&Phot. Chem., 2005, 23: 175 [史福强, 张 路, 安静仪, 赵 濉, 俞稼镛. 感光科学与光化学(Ganguangkexue Yu Guang Ниахие), 2005, 23: 175]
7 Tan, X. L.; Zhang, L.; Zhao, S.; Li, W.; Ye, J. P.; Yu, J. Y.; An, J. Y. Langmuir, 2004, 20: 7010

8 Wang, L. Ph. D. Dissertation. Beijing: Technical Institute of Physics Chemisry, Chinese Academy of Sciences, 2004 [王 琳. 博士学位论文. 北京: 中国科学院理化技术研究所, 2004]

9 Zhao, G. X. Principles of surfactants action. Beijing: China Light Industry Press, 2003: 704 [赵国玺. 表面活性剂作用原理. 北京: 轻工业出版社, 2003: 704]

10 Caponetti, E.; Triolo, R.; Patience, C. J. Colloid and Interface Sci., 1987, 116: 200

11 Rosen, M. J. Surfactants and interfacial plenomena. New Jersey: John Wiley and Sons, Inc., 2004: 145

\title{
Surface Properties of Sodium Alkylbenzenesulfonates with Additional Side Chains*
}

\author{
JIANG, Xiao-Ming ZHANG, Lu AN, Jing-Yi ZHAO, Sui YU, Jia-Yong \\ (Technical Institute of Physics and Chemistry, Chinese Academy of Sciences, Beijing 100101)
}

\begin{abstract}
The relationships between the structures of sodium alkylbenzensulfonates with additional side chains and surface properties, especially the influence of the side chains, were studied. From the surface tension measured in pure water and $0.171 \mathrm{~mol} \cdot \mathrm{L}^{-1} \mathrm{NaCl}$ solutions, the following parameters were calculated:the critical micelle concentration $(\mathrm{cmc})$, the surface excess concentration at surface saturation $\left(\Gamma_{\max }\right)$, the minimum area $\left(A_{\min }\right)$, the surface tension at the $\mathrm{cmc}\left(\gamma_{\mathrm{max}}\right)$, the effectiveness of a surfactant in reduing surface tension $\left(\pi_{\mathrm{cmc}}\right)$, the efficiency of a surfactant in reduing surface tension $\left(\mathrm{pC}_{20}\right)$, the standard free energy change upon adsorption $\left(\Delta G_{\mathrm{ad}}^{0}\right)$. The main chain and side chain have different effects on the surface properties. With increasing the number of carbons in the main chain or the side chains, cmc decreases, but the decrease degree of cmc is quite different, the main chain decreases cmc to a greater degree than the side chains do. While the number of carbons increases, the main chain increases $\Gamma_{\max }$, but the side chain decreases $\Gamma_{\max }$. These surface properties were explained by the structures of sodium alkylbonzensulfonate with additional side chains.
\end{abstract}

Keywords: Alkylbenzensulfonate, Molecular structure, Critical micelle concentration, Surface excess concentration 